\title{
Evaluation and Identification of Celiac Disease Associated Antigen DQ2 and DQ8 in Northern Indian-Kashmir Valley
}

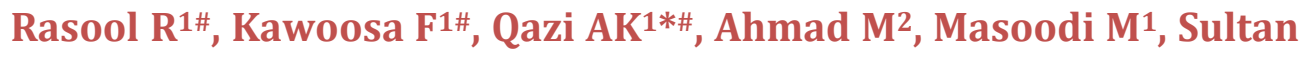 \\ $\mathrm{T}^{3}$, Beigh $\mathrm{AH}^{1}$ and Shah $\mathrm{ZA}^{1}$ \\ ${ }^{1}$ Department of Immunology and Molecular Medicine, Sher-I-Kashmir Institute of \\ Medical Sciences, India \\ ${ }^{2}$ Department of Gastroenterology, Sher-I-Kashmir Institute of Medical Sciences, India \\ ${ }^{3}$ Department of Clinical Biochemistry, University of Kashmir, India \\ \#Equally Contributed
}

\section{Research Article \\ Volume 3 Issue 1}

Received Date: May 18, 2019

Published Date: June 04, 2019

DOI: $10.23880 /$ jes- 16000119

*Corresponding author: Asif Khurshid Qazi, Department of Immunology and Molecular Medicine, Sher-I-Kashmir Institute of Medical Sciences, Soura, Srinagar, J\&K, India, Email: asifkqazi@gmail.com

\section{Abstract}

Background: Celiac disease (CD) is a commonly occurring food gluten sensitive enteropathy characterized by smallintestinal mucosal injury and nutrient malabsorption. It is known in genetically susceptible individuals by the ingestion of wheat gluten and similar proteins of other cereals, inducing an abnormal immune response. The genetic susceptibility of CD is associated with specific Major Histocompatibility Complex (MHC) II alleles that encode human leukocyte antigen (HLA) DQ2 or HLA DQ8 heterodimers.

Aim of Study: The present study was aimed at identification and evaluation of HLA "DQ2" and "DQ8" heterodimers in human subjects and determination of prevalence of Celiac disease in Kashmiri population.

Methodology: A total of sixty symptomatic clinical cases comprising of twenty five (25) males and thirty five (35) females were included in this study. The typing of HLA alleles was carried out by employing Sequence Specific Primers (SSP)-PCR.

Results: Our results demonstrated that among sixty participants, percentage of DQ2+/ DQ8+, DQ2+/DQ8-, DQ2-/DQ8+ and DQ2 / DQ8 were 11.66\%, 53.33\%, 8.33\% and 26.66\%. Moreover, among the twenty five male participants, five were $\mathrm{DQ}^{+} / \mathrm{DQ}^{+}$, ten DQ2+ $/ \mathrm{DQ8}$, two DQ2 / DQ8+, and eight DQ2 / DQ8- Similarly, among thirty five female participants, two were $\mathrm{DQ}^{+} / \mathrm{DQ}^{+}$, twenty two DQ2+/ DQ8, three DQ2 / DQ8+, and eight DQ2 / DQ8- Furthermore, our results also revealed that among the sixty participants, twelve (20\%) participants had low, fourteen $(23.33 \%)$ participants have high, eighteen (30\%) participants have very high and sixteen (26.67\%) participants have no susceptibility to celiac disease. In terms of gender predisposition we found that male subjects were $28 \%$ and females $45 \%$ susceptible to celiac disease. 


\section{Journal of Embryology \& Stem Cell Research}

Conclusion: From the current study we establish that majority of the subjects under study are susceptible to celiac disease and notably females are more susceptible as compared to males. Findings of the recent work could possibly aid in better understanding the prevalence of CD in Jammu and Kashmir. The high ratio of CD in females than males is also a question of future research.

Keywords: Celiac disease; Human Leukocyte antigen; Polymerase Chain Reaction; Gluten

Abbreviations: CD: Celiac Disease; MHC: Major Histocompatibility Complex; SSP: Sequence Specific Primers; EMA: Endomysia Antibodies; HLA: Human Leukocyte Antigen; DPG: Delaminated form of Gliadin Peptides; SKIMS: Sher-I-Kashmir Institute of Medical Sciences.

\section{Introduction}

Celiac disease (CD) also known as celiac sprue or gluten-sensitive enteropathy, is an immune modulated, chronic inflammatory disorder of the small bowel mucosa, which occurs in genetically predisposed individuals induced by the ingestion of gluten, the major storage protein of wheat and similar grains [1]. CD is now considered a relatively common disease worldwide and prevalence rate of approximately $1 \%$, ranging from 0.71 $1.25 \%$ with female preponderance in early age group has been reported. Contrary to earlier thoughts of low prevalence rates among Indian population, recent evidence shows an increasing prevalence even in India, especially North India currently its prevalence rate is approximately $0.6-1 \%$ [2]. In celiac disease, exposure to gluten, in genetically predisposed individuals, leads to the development of immune reactions associated with the activation of CD4, CD8 lymphocytes, production of proinflammatory cytokines, excessive proliferation of intraepithelial lymphocytes, migration of macrophages to the intestinal wall and intestinal mucosa besides that there is induction of $\beta$ lymphocytes, which produces specific antibodies to tissue transglutaminase 2 (tTG2), endomysia antibodies (EMA) and the delaminated form of gliadin peptides (DPG). This process leads to mucosal damage of the small bowel and other organs, producing symptoms ranging from malabsorption like diarrhoea, abdominal distension and weight loss, to nonspecific signs and symptoms such as fatigue, osteoporosis or iron deficiency anaemia [3]. The pathogenesis of CD involves interplay between genetic, epigenetic and immune factors. The major susceptibility genes in $\mathrm{CD}$ are the human leukocyte antigen (HLA)-DQ2 and HLA-DQ8, cluster which are necessary, but not sufficient to cause disease. The DQ genes account for approximately $40 \%$ of the genetic risk. Other genetic factors, including non-HLA genes that are involved in barrier and immune function, also contribute to disease susceptibility that accounts for a small proportion of the overall risk [4,5]. Till date no study has been carried out to evaluate and identify the the HLA DQ2 and HLA DQ8 haplotypes in kashmiri population with cealic disease.

\section{Materials and Methods}

Blood samples of 67 subjects, including 25 males and 35 females with CD diagnosed on the basis of clinical symptoms, serological investigations and small-intestine biopsy were collected in the Department of Immunology and Molecular Medicine, Sher-I-Kashmir Institute of Medical Sciences (SKIMS), which serves as a tertiary care referral centre for patients with known, suspected or complicated CD.

\section{DNA Extraction}

Isolation of whole genomic DNA from blood treated with anticoagulant was done using DNA Sure ${ }^{\circledR}$ Blood Mini kit. 20ul of proteinase $\mathrm{k}$ was added to remove protein fraction from $200 \mathrm{ul}$ blood sample. 200ul of lysis buffer was added to aid cell lysis followed by an incubation period of 10 mins at $52^{\circ} \mathrm{C}$. Further $200 \mathrm{ul}$ ethanol was added; the mixture was transferred to mini spin column tubes and centrifuged for 2 mins at maximum speed followed by two steps washing with wash buffers and centrifugation at maximum speed for 2 mins to remove any residual salt or proteins. Finally elution buffer was used to elude the pure DNA after allowing the buffer to stand in the tube for a few minutes before centrifugation. The concentration of the DNA obtained was measured by Bio spectrometer (Eppendorf) at wavelength of $260 \mathrm{~nm}$. The purity of DNA was checked by using $\mathrm{A}_{260} / \mathrm{A}_{280}$ ratio. 


\section{Journal of Embryology \& Stem Cell Research}

\section{HLA Haplotype by SSP PCR}

HLA testing was performed on DNA samples by using Sequence Specific Primers (SSP)-PCR. For this purpose, the HISTO TYPE SSP Kits from BAG Health Care, Germany were used. The HLA-DQ2 and DQ8 genotyping was done on the basis of the alleles such as

- DQA1*05, DQB1*02, DQA1*0301, and DQ B1*0302. The individuals having alleles

- DQ A1*05 and DQ B1*02 were considered to have DQ2 genotype and those having allele

- DQ A1*0301 and DQ B1*0302 were considered to have the DQ8 genotype.

Patients were considered to be positive for $C D$ enabling HLA genes if they carried the alleles that encode DQ2 and/or DQ8 genotype.

\section{Agarose Gel Electrophoresis}

The amplified products were detected and confirmed by comparing with a $100 \mathrm{bp}$ DNA marker ladder by electrophoresis on $2 \%$ agarose gel containing ethidium bromide in a mini gel system. Electrophoresis was carried out at a constant voltage of $130 \mathrm{~V}$ for 20 minutes. The gel was analysed on the gel doc [alpha imager EC].

\section{Results}

Reaction patterns revealed that among the sixty participants, seven $(11.66 \%)$ were DQ2+/ DQ8+, thirty two $(53.33 \%)$ were DQ2+/ DQ8-, five $(8.33 \%)$ were DQ2/ DQ8+, and sixteen (26.66\%) were DQ2-/ DQ8- (Figure 1).

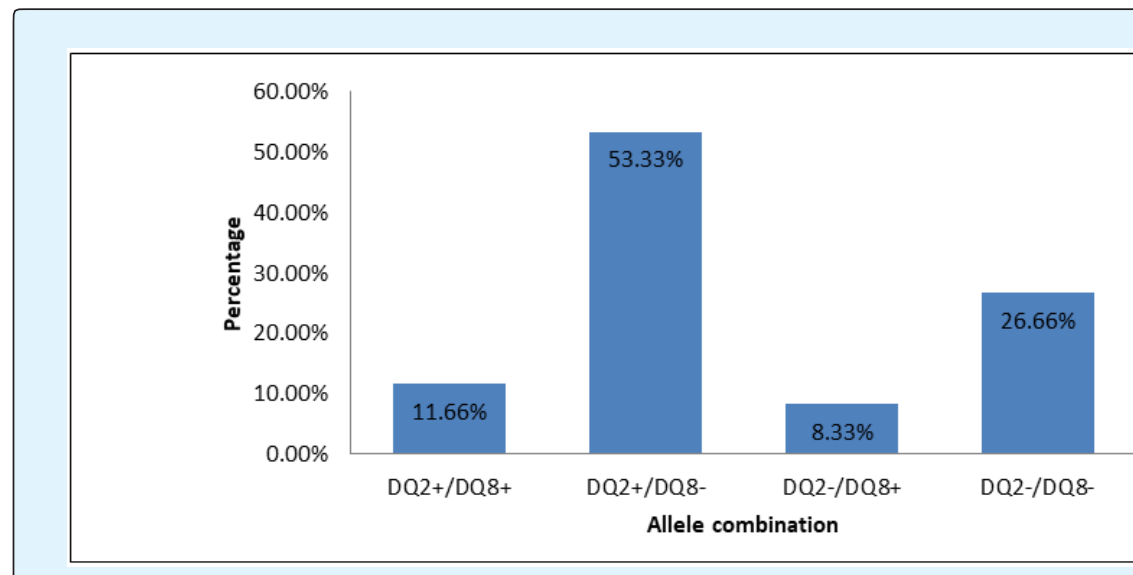

Figure1: Percentage distribution of different allele combinations.

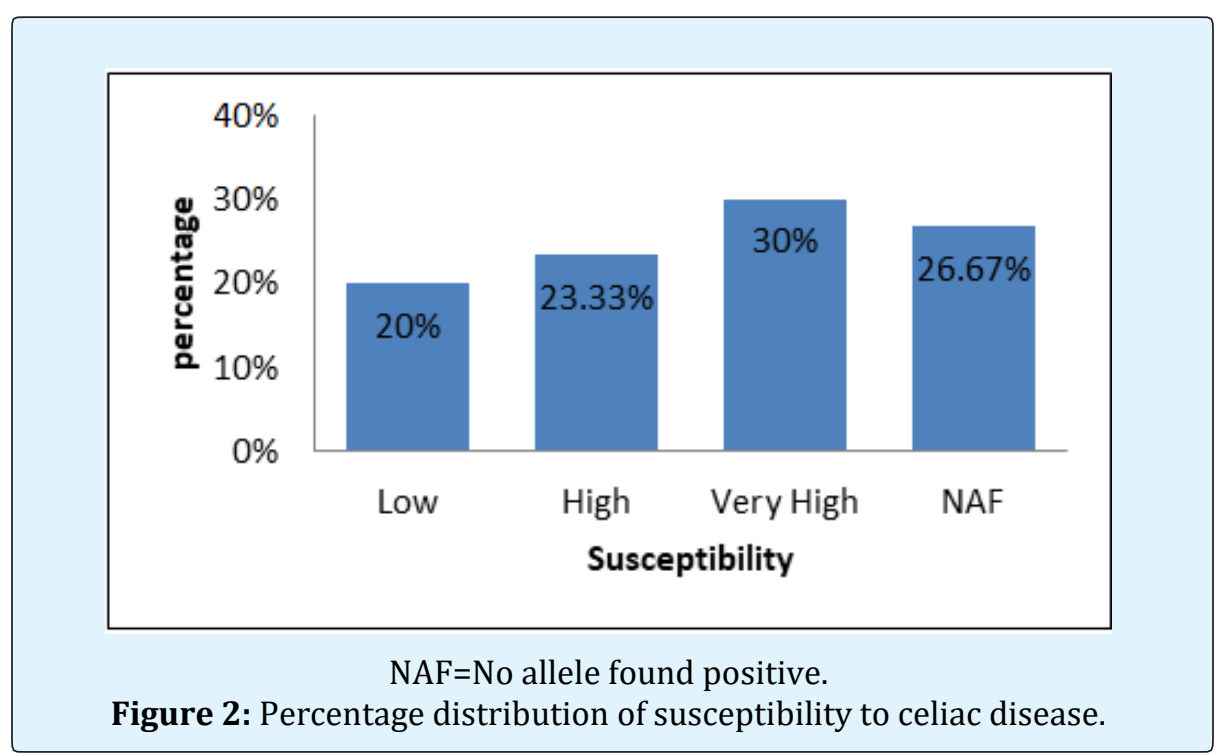

Qazi AK, et al. Evaluation and Identification of Celiac Disease Associated Antigen DQ2 and DQ8 in Northern Indian-Kashmir Valley. J Embryol Stem Cell 


\section{Journal of Embryology \& Stem Cell Research}

Results revealed that among the sixty participants, twelve (20\%) participants have Low, fourteen (23.33\%) participants have high, eighteen $(30 \%)$ participants have very high and Sixteen (26.67\%) participants have no susceptibility to celiac disease (Figure 2).

Results also revealed that among the twenty five male participants, five were $\mathrm{DQ}^{+}{ }^{+}$ DQ8 ${ }^{+}$, ten were $\mathrm{DQ}^{+} / \mathrm{DQ8}$, two were $\mathrm{DQ}_{2} \% \mathrm{DQ8}^{+}$, and eight were $\mathrm{DQ} 2 \% \mathrm{DQ8}$ pointing out that about $68 \%$ of the studied male participants were susceptible to celiac disease. Among the thirty five female participants, two were $\mathrm{DQ}^{+} / \mathrm{DQ}^{+}$, twenty two were $\mathrm{DQ}^{+} / \mathrm{DQ8}$, three were $\mathrm{DQ2} / \mathrm{DQ8}^{+}$, and eight were DQ2 / DQ8- indicating that $76.53 \%$ of the studied female participants were susceptible to celiac disease (Figure 3).

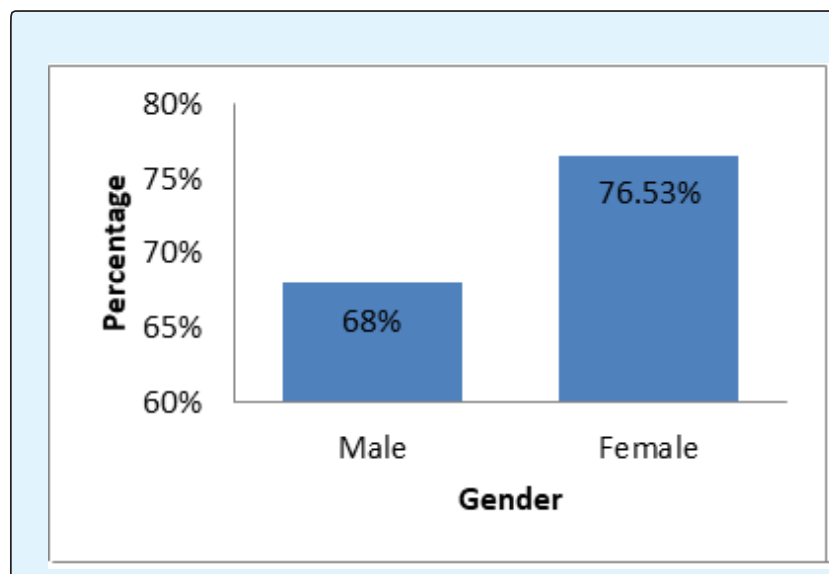

Figure3: Gender based percentage of celiac disease.

\section{Discussion and Conclusion}

The genetics of $C D$ is known to involve a number of factors, HLA DQ2 and DQ8 genes being the most important that are responsible for up to $40 \%$ of the genetic heritability of [6-9] more than $95 \%$ of celiac patients share the major histocompatibility complex II class human leukocyte antigen (HLA) DQ2 or DQ8 haplotype; patients negative for both types are unlikely to suffer from celiac disease [10]. The major genetic determinants are HLA-DQA1*05 and HLA-DQB $1 * 02$, which encode the DQ2 heterodimer. HLA-DQ2 (DQ2) is a serotype group within HLA-DQ (DQ) serotyping system. DQ8, the split antigen of the DQ3 broad antigen is encoded by an allele $\mathrm{B} 1 * 0302[6-8,10]$.

To date, very few studies have potentially investigated the prevalence of celiac disease in population-based samples. The prevalence of CD in the Indian subcontinent is still not clear but is likely to be progressively increasing [11]. During the recent years, it has been reported that in wheat- consuming states such as Punjab, Haryana, Delhi, Rajasthan, Uttar Pradesh, Bihar and Madhya Pradesh, prevalence of $\mathrm{CD}$ in predominantly higher on an epidemiological basis [12] studies on occurrence of celiac disease in Uttar Pradesh as well as from Delhi have indicated its similarity with the European susceptibility patterns $[13,14]$. The rising number of celiac disease cases in hospitalized patients has been reported from the Ludhiana over the last 10 years [15]. However, past studies indicate that there is difference in prevalence of celiac disease in the northern and southern part of India [11]. It is likely that celiac disease would be more common in North India [16]. Thus the regional difference of $\mathrm{CD}$ occurrence in India could be conceivably related to the genetic differences integrated with variation in staple diet pattern. However such reports from Jammu and Kashmir fall under grey area. Current study was conducted on the ethnic Kashmiri population and results of the current study revealed that among the participants, majority of individuals (73.33\%) were susceptible to celiac disease.

Current study included both male and female participants. The results highlighted that among the male participants about $68 \%$ were having susceptibility to celiac disease $\left(20 \%\right.$ were $\mathrm{DQ}^{+} / \mathrm{DQ} 8{ }^{+}, 40 \%$ were $\mathrm{DQ2}{ }^{+}$/ DQ8-, 8\% were DQ2-/ DQ8+, and 32\% were DQ2\%/ DQ8-). Among the female participants $76.53 \%$ were having susceptibility to celiac disease $5.71 \%$ were DQ2 ${ }^{+} / \mathrm{DQ}^{+}$, $62.86 \%$ were $\mathrm{DQ}^{+} / \mathrm{DQ} 8-8.57 \%$ were $\mathrm{DQ} 2 \% \mathrm{DQ}^{+}$, and $22.86 \%$ were DQ2-/ DQ8-). We found higher percentage of susceptibility in females compared to males. Our findings on gender based susceptibility are supported by many earlier studies which reported a higher proportion of females to be positive for celiac disease as compared to males [16-18].

In conclusion, current study indicated that majority of the individuals among studied participants were susceptible to celiac disease and remarkably females are more susceptible as compared to males. Finding of the recent work could possibly pave a way for better understanding of the prevalence of $\mathrm{CD}$ in Jammu and Kashmir. However, this is a preliminary study involving a limited number of samples, therefore further studies with larger sample size need to be carried out for further validation of the results. 


\section{Journal of Embryology \& Stem Cell Research}

\section{References}

1. Kagnoff MF (2005) Overview and pathogenesis of celiac disease. Gastroenterology 128(4): 10-18.

2. Fasano A, Catassi C (2012) Clinical practice. Celiac disease. N Engl J Med 367(25): 2419-2426.

3. Husby S, Koletzko S, Korponay Szabó IR, Mearin ML, Phillips A, et al. (2012) European Society for Pediatric Gastroenterology, Hepatology, and Nutrition guidelines for the diagnosis of coeliac disease. J Pediatr Gastroenterol Nutr 54(1): 136-160.

4. Gujral N, Freeman HJ, Thomson AB (2012) Celiac disease: Prevalence, diagnosis, pathogenesis and reatment. World Journal of Gastroenterology WJG 18(42): 6036-6059.

5. Kupfer SS, Jabri B (2012) Pathophysiology of celiac disease. Gastrointest Endosc Clin N Am 22: 639-660.

6. Bourgey M, Calcagno G, Tinto N, Gennarelli D, Margaritte Jeannin P, et al. (2007) HLA related genetic risk for celiac disease. Gut 56(8): 1054-1059.

7. Liu E, Rewers M, Eisenbarth GS (2005) Genetic testing: who should do the testing and what is the role of genetic testing in the setting of celiac disease? Gastroenterology 128(4): 33-37.

8. Margaritte Jeannin P, Babron MC, Bourgey M, Louka AS, Clot F, et al. (2004) HLA-DQ relative risks for coeliac disease in European populations: a study of the European Genetics Cluster on Coeliac Disease. Tiss Antig 63(6): 562-567.

9. Margaritte Jeannin $\mathrm{P}$, Babron $\mathrm{MC}$, Bourgey M, Louka AS, Clot F, et al. (2004) HLA-DQ relative risks for coeliac disease in European populations: a study of the European Genetics Cluster on Coeliac Disease. Tiss Antig 63(6): 562-567.
10. Kaukinen K, Partanen J, Mäki M, Collin P (2002) HLADQ typing in the diagnosis of celiac disease. Am J Gastroenterol 97: 695-699.

11. Deora NS, Deswal A, Dwivedi M, Mishra HN (2014) Prevalence of coeliac disease in India: A mini review. Int J Latest Res Sci Technol 3: 58-60.

12. Yachha SK (2006) Celiac disease: India on the global map. Journal of gastroenterology and hepatology 21(10): 1511-1513.

13. Agrawal S, Gupta A, Yachha SK, Müller Myhsok B, Mehrotra P, et al. (2000) Association of human leucocyte-DR and DQ antigens in coeliac disease: a family study. J Gastroenterol Hepatol 15(7): 771-774.

14. Kaur G, Sarkar N, Bhatnagar S, Kumar S, Rapthap CC, et al. (2002) Pediatric celiac disease in India is associated with multiple DR3-DQ2 haplotypes. Hum Immunol 63(8): 677-682.

15. Sood A, Midha V, Sood N, Kaushal V, Puri H (2001) Increasing incidence of celiac disease in India. AM J Gastroenterol 96(9): 2804-2815.

16. Makharia GK, Verma AK, Amarchand R, Bhatnagar S, Das P, et al. (2011) Prevalence of celiac disease in the northern part of India: a community based study. J Gastroenterol Hepatol 26(5): 894-900.

17. Bai D, Brar P, Holleran S, Ramakrishnan R, Green PH (2005) Effect of gender on the manifestations of celiac disease: evidence for greater malabsorption in men. Scand J Gastroenterol 40(2): 183-187.

18. Barada K, Bitar A, Mokadem MA, Hashash JG, Green P (2010)Celiac disease in Middle Eastern and North African countries: a new burden? World J Gastroenterol 16(12): 1449-1457. 